Artículo de investigación

\title{
ALMACENAMIENTO DE CARBONO ORGÁNICO EN SUELOS EN SISTEMAS DE PRODUCCIÓN DE CAFÉ (Coffea arabica L.) EN EL MUNICIPIO DEL LÍBANO, TOLIMA, COLOMBIA
}

\section{Storage of soil organic carbon in coffee (Coffea arabica L.) production systems in the municipality of Líbano, Tolima, Colombia}

Palabras clave: cambio climático, Cordia alliodora, densidad aparente, gases de efecto invernadero, sistemas agroforestales.

Key words: climate change, Cordia alliodora, bulk density, greenhouse gases, agroforestry systems.

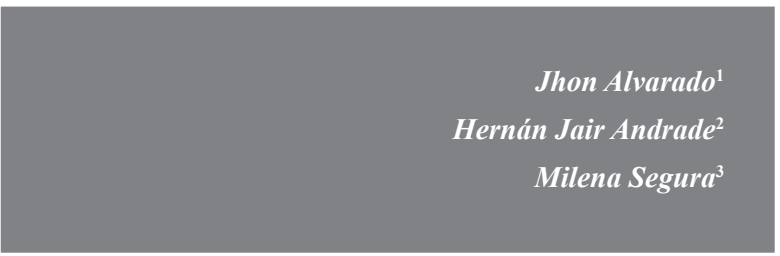

Sin embargo, el SAF con plátano tendió a tener menor DA que el monocultivo y SAF con nogal $\left(0.83 \pm 0.03\right.$ vs. $0.88 \pm 0.03$ vs. $0.92 \pm 0.04 \mathrm{~g} \cdot \mathrm{cm}^{-}$ ${ }^{3}$, respectivamente). Estos sistemas de producción almacenaron entre 50 y $54 \mathrm{t} \cdot \mathrm{ha}^{-1}$ de COS en los primeros $30 \mathrm{~cm}$ de profundidad, lo cual demuestra su capacidad de almacenamiento de carbono y potencial mitigación del cambio climático.

\section{ABSTRACT}

The increase in greenhouse gas emissions from anthropogenic sources has resulted in climate change, which affects all living beings. Coffee (Coffea arabica L.) plantations, in monoculture or together with timber species such as salmwood (Cordia alliodora), mitigate climate change due to fixation of atmospheric $\mathrm{CO}_{2}$ that is deposited in biomass and soils. This study was carried out in the municipality of Líbano, Tolima, Colombia with the objective of defining which of the following coffee production systems store more soil organic carbon (SOC): 1) monoculture, 2) agroforestry systems (AFS) with salmwood, and 3) AFS with plantain. Farms with those systems that are the most dominant in the study zone were selected. From each system, five repetitions were identified to be de producción no afectaron significativamente $(p>0.05)$ la DA ni la concentración de COS.

Grupo de Investigación Producción Ecoamigable de Cultivos Tropicales, Facultad de Ingeniería Agronómica, Universidad del Tolima. Ibagué, Colombia.jse_28@hotmail.com

Grupo de Investigación Producción Ecoamigable de Cultivos Tropicales, Facultad de Ingeniería Agronómica, Universidad del Tolima. Ibague, Colombia. hjandrade@ut.edu.co. Autor para correspondencia.

Grupo de Investigación Producción Ecoamigable de Cultivos Tropicales, Facultad de Ingeniería Forestal, Universidad del Tolima. Ibagué, Colombia.masegura@ut.edu.co 
analyzed with a completely randomized design. In each plot or repetition, five samples for bulk density (BD) using the cylinder method and a composite sample for concentration of SOC were taken and analyzed using the Walkley and Black approach. The systems of production did not significantly $(\mathrm{p}$ $>0.05$ ) affect either the BD or the concentration of SOC. However, AFS with plantain tended to have less BD than monoculture and AFS with salmwood $\left(0.83 \pm 0.03\right.$ vs $0.88 \pm 0.03$ vs $0.92 \pm 0.04 \mathrm{~g} \cdot \mathrm{cm}^{-3}$, respectively). These systems of production stored between 50 and $54 \mathrm{t} \cdot \mathrm{ha}^{-1}$ of SOC in the top $30 \mathrm{~cm}$, which indicates their capacity for climate change mitigation.

\section{INTRODUCCIÓN}

El calentamiento global es uno de los problemas ambientales más graves de la humanidad en este momento, el cual se manifiesta mediante variaciones en el clima. Esto se evidencia en los aumentos del promedio mundial de la temperatura del aire y del océano, el cual ha pasado de 0.6 a $0.74{ }^{\circ} \mathrm{C}$, y el derretimiento generalizado de hielo y nieve, reflejado en los datos satelitales, que muestran que el promedio anual de la superficie de los hielos marinos árticos se ha reducido en un $2.7 \%$ cada 10 años, lo cual provoca un aumento del nivel medio del mar mundial. Durante los últimos siglos se ha provocado un aumento en la emisión de gases de efecto invernadero (GEI) hacia la atmósfera, entre ellos el dióxido de carbono $\left(\mathrm{CO}_{2}\right)$ ha sido el más importante por su cantidad $\mathrm{y}$ efecto potencial en el calentamiento global. Las emisiones anuales de los GEI se incrementaron $80 \%$ entre 1970 y 2004 . Además, la disminución a largo plazo de las emisiones de $\mathrm{CO}_{2}$ por unidad de energía suministrada invirtió su tendencia a partir del año 2000 (IPCC, 2007).

La reducción de emisiones de GEI se puede lograr mediante los tres mecanismos propuestos por el Protocolo de Kioto: comercio de derechos de emisión, implementación conjunta y el mecanismo de desarrollo limpio (MDL) (Vargas \& Samayoa, 2009). El MDL está diseñado para promover la inversión en proyectos que capturen o disminuyan las emisiones de GEI en países en vía de desarrollo, por medio de la aforestación y reforestación. Es así como este mecanismo es uno de los que promueven las alianzas entre países desarrollados y en vía de desarrollo, de esta manera estas naciones colaboran mutuamente en obtener un beneficio común en el ámbito mundial a largo plazo, donde cada parte ayudará a mitigar la acumulación de GEI. Las plantaciones forestales y los sistemas agroforestales son una opción realista para incrementar el secuestro de carbono de agroecosistemas en biomasa y materia orgánica (Albrecht \& Kandji, 2003; Montagnini \& Nair, 2004; Oelbermann et al., 2004; Soto-Pinto et al., 2010).

La producción de café (Coffea arabica) en Colombia se realiza bajo varios sistemas de producción, tales como en monocultivo, bajo sombra con perennes como musáceas, o en sistemas agroforestales (SAF) con diferente estructura y composición de doseles. Estos últimos tienen en cuenta el componente leñoso perenne, que permite la fijación de carbono en la biomasa y después su incorporación al suelo (Farfán, 2007).

El objetivo de este estudio fue estimar el almacenamiento de carbono orgánico de suelos en los sistemas de producción de café más dominantes del municipio del Líbano, Tolima, Colombia. La hipótesis a probar es que el almacenamiento de carbono orgánico en suelo es mayor en sistemas agroforestales con nogal cafetero, que en sistemas agroforestales con plátano y en monocultivo. Los resultados de la estimación de carbono en dichos sistemas contribuyen al desarrollo de políticas para la implementación de sistemas de producción de café que ayuden a mitigar el cambio climático global.

\section{MATERIALES Y MÉTODOS}

\section{AREA DE ESTUDIO}

El estudio se desarrolló en el municipio del Líbano, en el norte del departamento del Tolima $\left(45^{\circ} 55^{\prime} \mathrm{N}\right.$ y $75^{\circ} 04^{\prime} \mathrm{O}$ ) en la zona de vida de bosque muy húmedo premontano (Holdridge, 1996): altitud aproximada de $1565 \mathrm{~m}$, precipitación media 
anual de $2235 \mathrm{~mm}$ y temperatura media de $20{ }^{\circ} \mathrm{C}$. Este municipio posee diversidad de sistemas de producción de café y es el segundo productor del grano en el departamento del Tolima (Federacafé, 2013). El Líbano limita con los municipios de: Villahermosa y Armero (norte), Murillo (oeste), Lérida y Santa Isabel (este) y Santa Isabel (sur) y se encuentra ubicado en el ecotopo $207 \mathrm{~B}, 4^{\circ}$ 47'$5^{\circ} 00^{\prime} \mathrm{N}$. El área de estudio está marcada por dos épocas de reducción de la precipitación: diciembreenero y julio-agosto, lo cual induce a un déficit hídrico. Asimismo, los suelos del área derivados de cenizas volcánicas son producto de las erupciones del complejo de volcanes Ruiz-Tolima (Lizcano et al., 2006). Además, presentan características particulares como buenas condiciones físicas, tales como la densidad aparente, la porosidad, estructura granular muy estable y alta capacidad de retención de humedad; a su vez, presentan una topografía ondulada de pendientes variables en cuanto a su grado y longitud (Fajardo, 2005).

\section{SELECCIÓN DE SISTEMAS DE PRODUCCIÓN Y MUESTRAS}

Se trabajó preliminarmente con una lista marco de productores de café proporcionada por el Comité de Cafeteros del Tolima, sede Líbano, a los cuales se entrevistó para identificar los sistemas de producción de café que manejaban en sus fincas y definir así los sistemas dominantes en la zona de estudio. Se eligieron fincas donde se encontraran los sistemas por estudiar, los cuales contrastan en tipo de sombra y manejo: 1) café en monocultivo, 2) café en sistemas agroforestales (SAF) con plátano (SAF-P; Musa AAB) y 3) café en SAF con nogal cafetero (SAF-N; Cordia alliodora). Se trabajó con un diseño experimental completamente al azar, con tres tratamientos (sistemas de producción de café) y cinco repeticiones.

\section{CONCENTRACIÓN DE CARBONO ORGÁNICO EN EL SUELO}

En cada unidad experimental se tomaron aleatoriamente 10 submuestras de suelos con un barreno helicoidal. Las muestras se tomaron entre $0 \mathrm{y}$ $30 \mathrm{~cm}$ de profundidad, debido a que en ella se concentra la mayor actividad de las raíces finas de cultivos (diámetro $>2 \mathrm{~mm}$ ) y, por ende, una mayor acumulación de materia orgánica por su senescencia (Andrade et al., 2008). Estas fueron homogenizadas hasta obtener una muestra compuesta, para estimar la concentración de carbono orgánico con el método de Walkley \& Black (1934) en el Laboratorio Laserex de la Universidad del Tolima.

\section{DENSIDAD APARENTE}

La densidad aparente se estimó empleando el método del cilindro de volumen conocido (Andrade \& Ibrahim, 2003), el cual consiste en tomar una muestra de suelo dentro de un anillo de metal de un volumen de $98.1 \mathrm{~cm}^{3}$, a una profundidad de 0-30 cm. Estas muestras fueron llevadas a peso constante, secando en horno a una temperatura de $105{ }^{\circ} \mathrm{C}$ en el Laboratorio de Fisiología Vegetal de la Universidad del Tolima. De cada unidad experimental se recolectaron cinco muestras para densidad aparente, la cual fue estimada mediante la ecuación 1.

$$
D a=\frac{P s s}{V c} \quad \text { Ec. } 1
$$

Donde $D a$ es la densidad aparente expresada en $\mathrm{g} \cdot \mathrm{cm}^{-3}, P s s$ es el peso de suelo seco en $\mathrm{g}$ y $V c$ es el volumen de cilindro $\left(98.1 \mathrm{~cm}^{3}\right)$

\section{CARBONO ORGÁNICO ALMACENADO EN EL SUELO}

Con base en el porcentaje de carbono orgánico y la densidad aparente se estimó el almacenamiento de carbono orgánico en el suelo con la metodología propuesta por Andrade \& Ibrahim (2003; ecuación 2) 
Donde COS es carbono orgánico de suelos (t tha $\left.{ }^{1}\right), P s$ es la profundidad del suelo $(\mathrm{cm}), D A$ es la densidad aparente $\left(\mathrm{g} \cdot \mathrm{cm}^{-3}\right)$ y $C O$ es la concentración de carbono orgánico en suelos $(\%)$

No se descontó la pedregosidad en el cálculo del almacenamiento de carbono en suelos, ya que en estos representa menos del $5 \%$ del volumen total del suelo.

\section{ANÁLISIS ESTADÍSTICO}

Se probó la normalidad de las variables estudiadas, mediante la prueba de Shapiro-Wilks modificada. Asimismo, se realizaron análisis de varianza a variables normales, mientras aquellas que no siguieron la normalidad se les realizó la transformación angular o arco seno, tal como el porcentaje de carbono orgánico del suelo (Steel \& Torrie, 1997). De igual forma, se realizaron pruebas de comparación de medias de Tukey para observar si existían diferencias estadísticas entre los tratamientos y las variables evaluadas. Igualmente, se llevó a cabo un análisis de correlación para observar la relación entre el almacenamiento de carbono, la densidad aparente y el porcentaje de carbono orgánico en el suelo. Finalmente, se empleó un análisis de regresión para encontrar relaciones matemáticas entre la densidad aparente y el porcentaje de carbono orgánico del suelo. Los análisis estadísticos fueron realizados con el programa InfoStat.

\section{RESULTADOS}

\section{DENSIDAD APARENTE DE SUELOS}

La densidad aparente de estos suelos fue baja (0.6 a $1.1 \mathrm{~g} \cdot \mathrm{cm}^{-3)}$, lo cual se esperaba para este tipo de suelos de origen volcánico. No se encontraron diferencias significativas $(p=0.61 ; F=0.51 ; g l$ $=12$ ) en la densidad aparente del suelo entre sistemas de producción de café; esto indica que los sistemas agroforestales ni el componente vegetal que crece junto al café no influyen sobre esta variable edáfica. El SAF-P presentó la menor densidad aparente promedio $\left(0.83 \pm 0.03 \mathrm{~g} \cdot \mathrm{cm}^{-3}\right)$, la cual fue $6 \%$ menor que la de los sistemas de café en monocultivo y $10 \%$ inferior a la de SAF-N (figura 1a).

\section{CONCENTRACIÓN DE CARBONO ORGÁNICO DEL SUELO}

El carbono orgánico del suelo se encontró a unas concentraciones que variaron entre 1.2 y $3.0 \%$ en estos suelos cafeteros. No se detectó un impacto significativo $(p=0.60 ; F=0.54 ; g l=12)$ de los sistemas de producción en la concentración de carbono orgánico en el suelo a $0-30 \mathrm{~cm}$. Sin embargo, la mayor acumulación se encontró en el

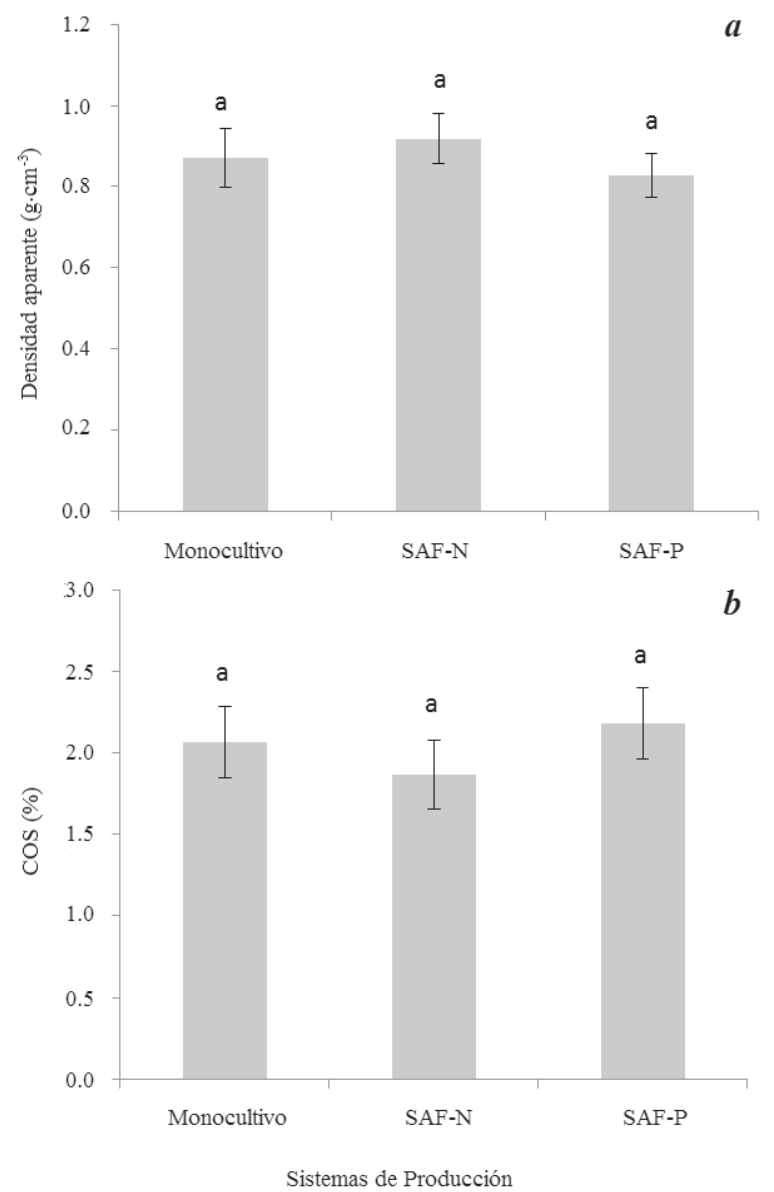

Figura 1. Densidad aparente $(\boldsymbol{a})$ y concentración de carbono orgánico $(\boldsymbol{b})$ en suelos a profundidad de 0 a $30 \mathrm{~cm}$ en sistemas de producción de café en el Líbano, Tolima, Colombia. SAF-N: sistemas agroforestales con nogal cafetero (Cordia alliodora); SAF-P: sistema agroforestal con plátano (Musa AAB). Las barras de error corresponden al error estándar. Letras diferentes indican diferencias estadísticamente significativas $(p>$ $0.05)$. 
SAF-P (2.2 \%), el cual fue 5 y $16 \%$ mayor que en monocultivo y en SAF-N, respectivamente (figura $1 b)$.

\section{RELACIÓN ENTRE LA DENSIDAD APARENTE Y LA CONCENTRACIÓN DE CARBONO ORGÁNICO DEL SUELO}

Se encontró una relación inversa entre la densidad aparente del suelo y el contenido de carbono orgánico $(r=-0.72 ; p<0.01$; figura 2$)$ a la profundidad estudiada; es decir, a medida que se incorpora carbono orgánico al suelo se disminuye su densidad aparente y, por ende, su compactación. Además, se desarrolló un modelo de regresión lineal para estimar la DA en función de la concentración de COS en estos suelos en los primeros $30 \mathrm{~cm}$. De esta manera se encontró una pendiente negativa $(-0.209)$, la cual indica la relación inversa entre la variables (figura 2).

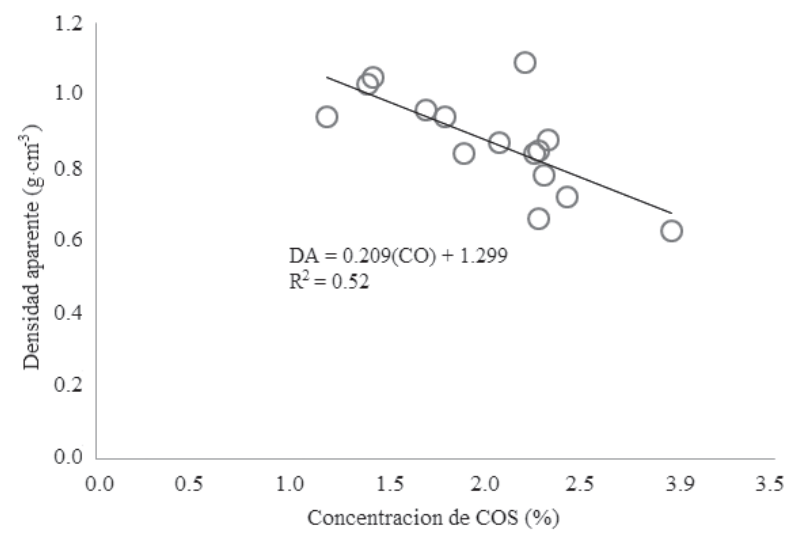

Figura 2. Relación entre la densidad aparente (DA) y el porcentaje de carbono orgánico en suelos (COS) a un profundidad de 0-30 $\mathrm{cm}$ en sistemas de producción de café (monocultivo y sistemas agroforestales con Cordia alliodora y Musa AAB) del municipio del Líbano, Tolima, Colombia.

\section{ALMACENAMIENTO DE CARBONO ORGÁNICO DE SUELOS}

Los suelos cafeteros evaluados tienen un alto almacenamiento de carbono, el cual se ubicó entre 33.6 y 72.3 t C.ha ${ }^{-1}$. El carbono orgánico almacenado en los primeros $30 \mathrm{~cm}$ de suelo no presentó diferencias estadísticas $(p=0.84 ; F=$ $0.18 ; g l=12$ ) entre sistemas de producción de café, a pesar de existir un rango de COS de $3.4 \mathrm{t} \mathrm{C} \cdot \mathrm{ha}^{-}$ ${ }^{1}$ entre tratamientos. El sistema de producción de café que mayor acumulación de COS presentó fue el café en monocultivo, seguido del SAF-P y, por último, el SAF-N ( $53.3 \pm 4.3$ vs. $52.8 \pm 4.3$ vs. 49.9 $\pm 4.3 \mathrm{t} \mathrm{C} \cdot \mathrm{ha}^{-1}$, respectivamente; figura 3 ).

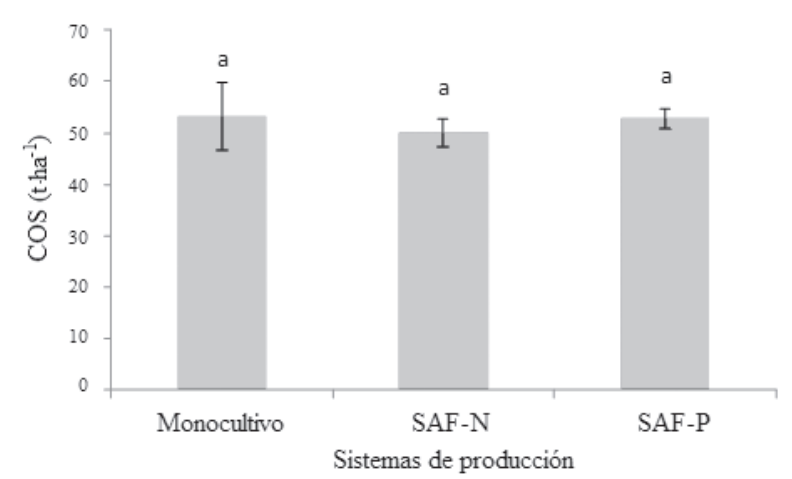

Figura 3. Carbono orgánico almacenado en suelos a una profundidad de 0-30 $\mathrm{cm}$ en sistemas de producción de café en el Líbano, Tolima, Colombia. SAF-N: sistemas agroforestales con nogal cafetero (Cordia alliodora); SAF-P: sistema agroforestal con plátano (Musa AAB). Las barras de error corresponden al error estándar. Letras diferentes indican diferencias estadísticamente significativas $(p<0.05)$.

\section{DISCUSIÓN}

La densidad aparente encontrada en la mayoría de plantaciones de café evaluadas es óptima para el establecimiento de estos sistemas de producción, debido a que valores entre 0.7 y $0.9 \mathrm{~g} \cdot \mathrm{cm}^{-3}$ no afectan el crecimiento del sistema radicular de los cultivos y más bien favorecen la utilización de actividades agrícolas que reducen la posibilidad de compactarlos y erosionarlos (Alvarado \& Forsythe, 2005). Inclusive, hay parcelas en las cuales la densidad aparente baja a $0.6 \mathrm{~g} \cdot \mathrm{cm}^{-3}$, lo cual trae grandes ventajas para la aireación, el movimiento del agua y el flujo de nutrientes (Fassbender, 1993). Arshad \& Martin (2002) incluyen a la densidad aparente como un indicador de la calidad de suelos, por su importancia en la penetración radicular, porosidad y análisis ajustados a una base volumétrica.

Igualmente, la densidad aparente encontrada en los SAF coincidió con lo reportado por Lizcano 
et al. (2006) en suelos colombianos derivados de cenizas volcánicas $\left(0.56\right.$ y $\left.1.03 \mathrm{~g} \cdot \mathrm{cm}^{-3}\right)$. Asimismo, Paz et al. (2006) encontraron densidades aparente entre 0.6 y $0.7 \mathrm{~g} \cdot \mathrm{cm}^{-3}$ en dos sistemas de sombrío de café, las cuales son ligeramente inferiores a lo encontrado en el Líbano, Tolima, Colombia. Los resultados de este estudio y de otros afirman que valores inferiores a $1 \mathrm{~g} \cdot \mathrm{cm}^{-3}$ son característicos de suelos orgánicos, mientras que valores inferiores a $0.9 \mathrm{~g} \cdot \mathrm{cm}^{-3}$ son propios de suelos ricos en alófana; es decir, andisoles. Estos estudios recalcan la importancia de la génesis de suelos en su DA y otras variables físicas dependientes.

Aunque no se encontraron diferencias significativas en densidad aparente entre sistemas, se puede apreciar una tendencia en la disminución de esta variable en el SAF-P. Esto puede ser debido a que el ciclo vegetativo del plátano es más corto y su fuste es herbáceo, lo cual permite una velocidad de descomposición de necromasa mayor, acelerando así la incorporación de materia orgánica al suelo y reduciendo la compactación del suelo. Otra explicación para la menor densidad aparente en SAF-P es la configuración del sistema radicular del plátano, el cual está conformado principalmente de raíces finas (diámetro $<2 \mathrm{~mm}$; Aránzazu et al., 2005), mayormente en los primeros $30 \mathrm{~cm}$ de profundidad del suelo. Esta reducción en densidad aparente promueve que los suelos sean más sueltos, incrementa el crecimiento y exploración de raíces, facilita la aireación, la movilidad del agua y la toma de nutrientes por las plantas. Adicionalmente, se ha encontrado que el plátano no afecta notablemente la producción de café (van Asten et al., 2011).

La concentración de carbono orgánico en estos suelos contrasta con lo encontrado para café a pleno sol y a sombrío parcial de guamo (Inga spp.) o de plátano (Musa AAB): 7.0 y $7.1 \%$, respectivamente (Sadeghian, 2011). Este mismo autor (Sadeghian, 2009) reportó un $9.6 \%$ de carbono orgánico en fincas del Líbano, Tolima. El COS es un indicador indirecto de la calidad de los suelos, principalmente desde el punto de vista físico y biológico, ya que está íntimamente relacionado con la materia orgánica. Las diferencias encontradas entre estudios podrían ser explicadas por la naturaleza de los suelos, principalmente debido a su origen. En el campo del servicio ambiental de la fijación de carbono atmosférico, un cambio positivo en la COS indica secuestro, mientras que una reducción de esta variable causa una emisión de $\mathrm{CO}_{2}$ a la atmósfera, tal como ha sido reportado en otros estudios (Chen et al. 2006; Andrade et al. 2008; Polyakov \& Lal 2008; Guntiñas et al. 2009).

La relación inversa entre la DA y el COS encontrada en esta investigación coincide con lo encontrado por Arvidsson (1998). Se observa así la importancia de la incorporación de materia orgánica al suelo, en primer lugar, para el mejoramiento de las propiedades físicas $\mathrm{y}$, en segundo lugar, para mejorar las condiciones químicas y biológicas. Este material vegetal, al depositarse en el suelo, es objeto de procesos de descomposicion o degradacion por parte de los microorganismos, hasta llegar a los componentes elementales (Fassbender, 1993; Martínez et al., 2008).

Aun cuando en este estudio no se evaluó la entomofauna, autores como Lal (2004) señalan que los depósitos de carbono en el suelo y su dinámica se encuentran estrechamente relacionados con la fauna edáfica, la cual es considerada un factor de gran influencia en las propiedades físicas y biológicas del suelo, especialmente en lo relacionado con la estructura, porosidad, aireación, infiltración, drenaje, ciclaje de nutrientes y flujo de materia orgánica. Asimismo, el incremento y diversidad de entomofauna está dado por la profundidad del suelo y se relaciona directamente con la concentración de COS y aporte de necromasa por parte de los sistemas de uso del suelo (Carvajal et al., 2009).

La estrecha e inversa relación entre la DA y el porcentaje de carbono orgánico del suelo indica que al incrementarse la concentración del COS en $1 \%$ se reduce la densidad en un poco más de $0.2 \mathrm{~g} / \mathrm{cm}^{3}(p<0.01)$. Estos cambios significativos producen grandes mejoras en la calidad del suelo, principalmente en el movimiento de agua y nutrientes en el perfil. Martínez et al. (2008) afirman que el carbono orgánico del suelo tiene un efecto importante en la agregación de las 
partículas del suelo, lo cual redunda en cambios en la porosidad, densidad aparente, retención de agua y de aire. Esto refuerza la importancia de la fijación de carbono orgánico en suelos tanto para la mitigación del cambio climático global como para el mejoramiento de suelos como sostén de la producción agropecuaria (Fassbender, 1993; Albrecht \& Kandji, 2003; Montagnini \& Nair, 2004; Oelbermann et al., 2004). La baja explicación del modelo $\left(R^{2}=0.52\right)$ sugiere que existen otros factores, además de la concentración del COS, que impactan a la DA y el secuestro de carbono en suelos. En estos factores se pueden incluir el origen y el material parental de estos suelos.

Los valores encontrados en este estudio, en promedio $52.0 \mathrm{t} \mathrm{C} \cdot \mathrm{ha}^{-1}$, fueron muy inferiores a lo reportado como promedio mundial para los andisoles en los primeros $30 \mathrm{~cm}\left(114 \mathrm{t} \mathrm{C} \cdot \mathrm{ha}^{-1}\right.$; Batjes, 1996). Esto podría estar indicando que los suelos han sido manejados de manera inadecuada en el pasado, lo cual ha causado pérdidas de COS y posibles emisiones de $\mathrm{CO}_{2}$ a la atmósfera. Sin embargo, los resultados aquí presentados sugieren que en cafetales, las especies vegetales acompañantes, tal como los árboles de nogal cafetero y las plantas de plátano, no afectan el almacenamiento de carbono orgánico del suelo $(p>0.05)$. Esto contrasta notablemente con lo reportado por Hergoualc'h et al. (2012), quienes encontraron un mayor almacenamiento de carbono en SAF con Inga densiflora en comparación a cafetales en monocultivo en los $10 \mathrm{~cm}$ superiores del suelo luego de siete años de establecido (25.2 vs. $9.8 \mathrm{t} \mathrm{C} \cdot \mathrm{ha}^{-1}$, respectivamente). Los resultados contrastantes pueden deberse a diferencias en la historia de uso del suelo, pues es posible que antes de estos cafetales se tuvieran otros sistemas de uso del suelo de mayor reducción de carbono orgánico del suelo.

El alto de contenido de COS promedio en el café monocultivo puede estar influenciado por la densidad de plantas de cafeto y, principalmente, el manejo adecuado de la plantación: podas, prácticas de conservación de suelo que permiten mayor acumulación de carbono en el perfil del suelo. El incremento del COS se debe principalmente a la caída de hojarasca y mortalidad y exudación de las raíces finas, lo cual depende de la distribución y actividad de las raíces. Al respecto hay que considerar que las raíces finas (diámetro $<2 \mathrm{~mm}$ ) son la principal fuente de acumulación de COS y en el caso de arbustos y herbáceas, como el plátano, se encuentran mayoritariamente en los primeros $30 \mathrm{~cm}$ de suelo. Siles et al. (2010) encontraron que el $75 \%$ de las raíces finas de un perfil de suelo de $1 \mathrm{~m}$ fue encontrado en los primeros 60 $\mathrm{cm}$. Investigadores han encontrado tendencias similares al evaluar la dinámica del COS en otros sistemas de producción en el trópico, tal como las pasturas (De Camargo et al., 1999; Fisher et al., 2004; Ibrahim et al., 2007; Maia et al., 2009).

La materia orgánica es un indicador clave de la calidad del suelo y de las funciones ambientales, entre ellas la fijación de carbono atmosférico; es decir, la actividad biológica que esta genera tiene gran influencia sobre las propiedades químicas y físicas del suelo (Suárez, 2002). Los valores encontrados en este estudio fueron inferiores a los obtenidos por Ávila et al. (2001) en SAF de café con Eucalyptus deglupta y poró (Erythrina poepiggiana $)$ a diferentes edades $\left(66.2-87.3 \mathrm{tC} \cdot \mathrm{ha}^{-1}\right.$ en $0-30 \mathrm{~cm}$ de profundidad). En contraste, Connolly \& Corea (2007) reportaron un almacenamiento de carbono orgánico en el suelo muy superior al presente y mencionado estudio (142.8 t C $\cdot \mathrm{ha}^{-1}$ en 0-30 $\mathrm{cm}$ de profundidad) en SAF con café en Nicaragua.

Ruiz \& Somarriba (2002) evaluaron el carbono en SAF en Nicaragua, ellos observaron que esos suelos almacenaban entre 42.3 y $120.9 \mathrm{t} \mathrm{C} \cdot \mathrm{ha}^{-1}$, con lo cual se obtuvo un rango más amplio. Estos autores recalcan que la variación de estos valores depende de variables como el uso del suelo, topografía y condiciones climáticas. Entretanto, Carvajal et al. (2009) encontraron un rango de $37-43 \mathrm{t} \mathrm{C} \cdot \mathrm{ha}^{-1}$ en los primeros $30 \mathrm{~cm}$ en paisajes andinos colombianos a una altitud similar a la de este estudio. De esta manera, se puede observar que los resultados obtenidos en esta investigación son congruentes con los reportados por otros autores. 
Corral et al. (2006) encontraron almacenamientos de carbono dentro del rango encontrado en este estudio en dos zonas agroecológicas del litoral ecuatoriano: $66.9,72.0$ y $78.8 \mathrm{t} \mathrm{C} \cdot \mathrm{ha}^{-1}$ en SAF con café y pachaco (Schizolobium parahybum), café y guadua, y café y nogal cafetero, respectivamente. En SAF con cacao, estos mismos autores encontraron un rango de almacenamiento de carbono orgánico en suelos de $70.7-81.1 \mathrm{t} \mathrm{C} \cdot \mathrm{ha}^{-1}$. Estos valores son superiores a los encontrados en el presente estudio. En Talamanca, Costa Rica, Andrade et al. (2008) estimaron en 48.8 y $61.7 \mathrm{t} \mathrm{C} \cdot \mathrm{ha}^{-1}$ el COS almacenado en SAF con banano y cacao, respectivamente. Estos resultados son comparables a lo obtenido en esta investigación.

El no encontrar diferencias estadísticas entre sistemas en este estudio probablemente se debió a que el mayor componente de raíces finas en estos sistemas es precisamente del café, común a los tres sistemas de producción evaluados. Es claro que a mayor productividad biológica del sistema ocurre un mayor contenido de carbono, por lo tanto, los agroecosistemas pueden tener mayor capacidad de fijar carbono que otros sistemas naturales e incluso que los bosques tropicales maduros (Rodríguez et al., 2009).

La fertilización nitrogenada inorgánica en estos sistemas de producción podría reducir la relación $\mathrm{C} / \mathrm{N}$, al aumentar el $\mathrm{Ny}$ no afectar significativamente el $\mathrm{C}$; de esta manera se produce una mayor biomasa y potencialmente mayor fijación de carbono orgánico en suelos (Novelli et al., 2009). Es importante considerar que las variaciones en el almacenamiento de COS obedecen a la influencia de factores edafoclimáticos que inciden en la acumulación de contenidos de materia orgánica.

\section{CONCLUSIONES}

La baja densidad aparente de estos suelos cafeteros está indicando unas condiciones edáficas óptimas para la producción de café. El incremento del almacenamiento de COS también parece tener otros impactos positivos en el suelo, ya que se reduce la DA, lo cual trae consecuencias favorables para la circulación de aire, agua y nutrientes en solución.
De acuerdo a esto, sistemas que incrementen la fijación de carbono atmosférico contribuirían, de igual manera, al mejoramiento de la calidad del suelo.

Los sistemas de producción de café en el municipio del Líbano, Tolima, Colombia, podrían fijar carbono atmosférico en los suelos con la potencial mitigación del cambio climático; sin embargo, los cafetales en monocultivo parecer ser levemente mejores para el incremento de la fijación de carbono orgánico en el suelo. No obstante, se debe considerar que los cafetales en monocultivo almacenan menos carbono en biomasa y afectan negativamente otros servicios ambientales, como aquellos derivados de la conservación de biodiversidad. La sombra asociada al café parece no contribuir a cambiar la densidad aparente del suelo en los primeros $30 \mathrm{~cm}$.

Aunque en este estudio no se detectó un impacto de los sistemas de producción en el almacenamiento de carbono orgánico del suelo, diversos autores han encontrado que los cafetales son una opción de mitigación del cambio climático en países tropicales. Estos sistemas acumulan COS, el cual puede ser más estable que otros componentes. Sin embargo, si se incluyen otros componentes como la biomasa arriba y abajo del suelo, el atractivo de estos sistemas para proyectos de venta de certificados de carbono, como los MDL o mercados voluntarios, se incrementaría notablemente.

\section{AGRADECIMIENTOS}

A todos los productores de café que apoyaron el estudio participando activamente en la toma de muestras de suelos en sus predios. Al Comité de Cafetero del Tolima por su apoyo en el contacto con los productores de café. Al Comité Central de Investigaciones de la Universidad del Tolima al apoyar el Proyecto Huella de Carbono en Sistemas de Producción de Café en el Municipio del Líbano, Tolima, código 80111. A Diana Skarly Canal, asistente del Laboratorio de Fisiología Vegetal y estudiante de MSc en ciencias biológicas de la Universidad del Tolima por su apoyo en campo y en laboratorio. 


\section{REFERENCIAS BIBLIOGRÁFICAS}

Albrecht, A., \& Kandji, S.T. (2003). Carbon sequestration in tropical agroforestry systems. Agriculture, Ecosystems and Environment, 99, 15-27.

Alvarado, A., \& Forsythe, W. (2005). Va-riaciones de la densidad aparente en órdenes de suelos de Costa Rica. Agronomía Costarricense, $29(00), 85-94$.

Andrade, H., \& Ibrahim, M. (2003). ¿Cómo monitorear el secuestro de carbono en los sistemas silvopastoriles? Agroforestería en las Américas, 10(39-40), 109-116.

Andrade, H.J., Brook, R., \& Ibrahim, M. (2008). Growth, production and carbon sequestration of silvopastoral systems with native timber species in the dry lowlands of Costa Rica. Plant and Soil, 308 (1-2), 11-22.

Aránzazu, H., Valencia, M., Arcila, P., Arias, C., Bolaños, B., \& Castellanos, C. (2005). Cultivo de plátano. Manual técnico del cultivo de plátano. Manizales, Colombia: Editores S.A. 124 p.

Arvidsson, J. (1998). Influence of soil texture and organic matter content on bulkdensity, air content, compression index and crop yield in field and laboratory compression experiments. Soil and Tillage Research, $49,159-170$.

Ávila, G., Jiménez, F., Beer, J., Gómez, M., \& Ibrahim, M. (2001). Almacenamiento, fijación de carbono y valoración de servicios ambientales en sistemas agroforestales en Costa Rica. Agroforestería en las Américas, 8(30), 32-35.

Arshad, M.A. \& Martin, S. (2002). Identifying critical limits for soil quality indicators in agro-ecosystems. Agriculture, Ecosystems and Environment, 88, 153-160.
Batjes, N.H. (1996). Total carbon and nitrogen in the soils of the World. European Journal of Soil Science, 47, 151-163.

Carvajal, A., Feijoo, A., Quintero, H., \& Rondón, M. (2009). Carbono orgánico del suelo en diferentes usos del terreno de paisajes andinos colombianos. Revista de la ciencia del suelo y nutrición vegetal, 9(3), 222-235.

Chen, Y., Wu, C.Y., Shui, J.G., \& Wang, J.Y. (2006). Emission and fixation of $\mathrm{CO}_{2}$ from soil system as influenced by long-term application of organic manure in paddy soils. Agricultural Sciences in China, 5(6), 456-461.

Connolly, R., \& Corea, C. (2007). Cuantificación de la captura y almacenamiento de carbono en sistema agroforestal y forestal en seis sitios de cuatro municipios de Nicaragua. Universidad Nacional Agraria, Managua, Nicaragua. $72 \mathrm{p}$.

Corral, R., Duicela, L.A., \& Maza, H. (2006). Fijación y almacenamiento de carbono en sistemas agroforestales con café arábico y cacao, en dos zonas agroecológicas del litoral ecuatoriano. X Congreso Ecuatoriano de la Ciencia del Suelo, "Avances del Conocimiento para la preservación del suelo". Guayaquil, Noviembre 22-24 del 2006. $15 \mathrm{p}$.

De Camargo, P.B., Trumbore, S.E., Martinelli, L.A., Davidson, E.A., Nepstad, D.C., \& Victoria, L. (1999). Soil carbon dynamics in regrowing forest of Eastern Amazonia. Global Change Biology, 5, 693-702.

Fajardo, N. (2005). El suelo. Uso y Manejo de Suelos. (49-75). Ibagué, Colombia: Litoimagen impresores. $315 \mathrm{p}$.

Farfán, F.F. (2007). Producción de café en sistemas agroforestales. En: Arcila, J., Farfán, F., Moreno, A., Salazar, L.F., Hincapié, 
E. (eds.). Sistemas de producción de café en Colombia. Cenicafé, Chinchiná, Colombia, 161-200.

Fassbender,H.W. (1993). Modelos edafológicos de sistemas agroforestales. $2^{a}$ edición. Centro Agronómico Tropical de Investigación y Enseñanza (CATIE), Turrialba, Costa Rica. 530 p.

Federacafé (Federación Nacional de Cafeteros de Colombia). (2013). Tolima café de alta calidad. Recuperadoel 1 defebrerode $2013 \mathrm{de}$ http://tolima.federaciondecafeteros.org/

Fisher, M.J., Rao, I.M., \& Thomas, R.J. (2004). Implications of land use change to introduced pastures on carbon stocks in the central lowlands of tropical South America. Environment, Development and Sustainability, 6, 111-131.

Guntiñas, M.E., Gil-Sotres, F., Leiro, M.C., \& Trasar-Cepeda, C. (2009). $\mathrm{CO}_{2}$ emission from soils under different uses and flooding conditions. Soil Biology \& Biochemistry, 41, 2598-2601.

Hergoualc'h, K., Blanchart, E., Skiba, U., Hénault, C., \& Harmand, J.M. (2012). Changes in carbon stock and greenhouse gas balance in a coffee (Coffea arabica) monoculture versus an agroforestry system with Inga densiflora, in Costa Rica. Agriculture, Ecosystems \& Environment, 148(15), 102-110.

Holdridge, L. (1996). Ecología basada en zonas de vida. $4^{\mathrm{a}}$ reimpresión, San José, Costa Rica, Instituto Interamericano de Cooperación para la agricultura, 1996, c1978. Colección Libros y Materiales Educativos/IICA; no. 83. $216 \mathrm{p}$.

Ibrahim, M., Chacón, M., Cuartas, C., Naranjo, J., Ponce, G., Vega, P., Casasola, F., \& Rojas, J. (2007). Almacenamiento de carbono en el suelo y la biomasa aérea en sistemas de uso de la tierra en paisajes ganaderos de Colombia, Costa Rica y Nicaragua. Agroforestería en las Américas, $45,27-36$.

IPCC (Intergovernmental Panel on Climate Change). (2007). Cambio climático 2007 -Informe de síntesis. Contribución de los Grupos de trabajo I, II y III al Cuarto Informe de evaluación del Grupo Intergubernamental de Expertos sobre el Cambio Climático [Equipo de redacción principal: Pachauri, R.K. y Reisinger, A. (directores de la publicación)]. IPCC, Ginebra, Suiza, 104 p.

Lal, R. (2004). Soil carbon sequestration to mitigate climate change. Geoderma, 123, $1-22$.

Lizcano, A., Herrera, M. C., \& Santamarina, J.C. (2006). Suelos derivados de cenizas volcánicas en Colombia. Revista Internacional de Desastres Naturales, Accidentes e Infraestructura Civil, 6(2), 167-198.

Maia, S.M.F., Ogle, S.M., Cerri, C.E.P., \& Cerri, C.C. (2009). Effect of grassland management on soil carbon sequestration in Rondônia and MatoGrosso states, Brazil. Geoderma, 149, 84-91.

Martínez, E., Fuentes, J. P., \& Acevedo, E. (2008). Carbono orgánico y propiedades del suelo. R. C. Suelo Nutr. Veg, 8(1), 6896.

Montagnini, F., \& Nair, P.K.R. (2004). Carbon sequestration: an underexploited environmental benefit of agroforestry systems. Agroforestry Systems, 61-62(1-3), 281295.

Novelli, L., Melchiori, R., \& Cavigli, O. (2009). Efecto de la fertilización nitrogenada so- 
bre el contenido de $\mathrm{c}$ y $\mathrm{n}$ en diferentes tamaños de agregados. Revista Científica Agropecuaria, 13(1-2), 25-32.

\section{Oelbermann, M., Voroney, R.P., \& Gordon, A.M.} (2004). Carbon sequestration in tropical and temperate agroforestry systems: a review with examples from Costa Rica and Southern Canada. Agriculture Ecosystems and Environment, 104, 359-377.

Paz, E., Sánchez, M., \& Sadeghian, S. (2006). Relación entre dos sistemas de sombrío de café y algunas propiedades del suelo en la meseta de Popayán, Colombia. Acta Agronómica, 55(4), 1-6.

Polyakov, V.O., \& Lal, R. (2008). Soil organic matter and $\mathrm{CO}_{2}$ emission as affected by water erosion on field runoff plots. Geoderma, 143, 216- 222.

Rodríguez, J.M., Camargo, J. C., Niño, J., Pineda, A.M., Arias, L. M., Echeverry, M.A., \& Miranda, C.L. (eds). (2009). Valoración de la Biodiversidad en la Ecorregión del Eje Cafetero. CIEBREG. Pereira, Colombia. 238 p

Ruiz, F., \& Somarriba A. (2002). Evaluación del efecto de la práctica de no quema en el almacenamiento de carbono y la fertilidad de suelos en las comunidades de las cámaras y sabana larga, Estelí, Nicaragua. Universidad centro americana. Managua, Nicaragua. 56 p.

Sadeghian, S. (2009). Calibración de análisis de suelo para $\mathrm{N}, \mathrm{P}, \mathrm{K}$ y $\mathrm{Mg}$ en cafetales al sol y bajo semisombra. Cenicafé, 60 (1), 7-24.

Sadeghian, S. (2011). Respuesta de cafetales al sol y bajo semisombra a nitrógeno y su relación con la materia orgánica del suelo. Rev. Fac. Nal. Agr. Medellín, 64(1), 57815791.
Siles, P., Harmand, J.M., \& Vaast, P. (2010). Effects of Inga densiflora on the microclimate of coffee (Coffea arabica $\mathrm{L}$.) and overall biomass under optimal growing conditions in Costa Rica. Agroforest. Syst, 78, 269-286.

Soto-Pinto, L., Anzueto, M., Mendoza, J., Jiménez Ferrer, G., \& de Jong, B. (2010). Carbon sequestration through agroforestry in indigenous communities of Chiapas, Mexico. Agroforestry Systems, 78, 39-51.

Steel, R., \& Torrie, J. (1997). Bioestadística principios y procedimientos. México: McGraw-Hill / Interamericana de México S.A. 622 p.

Suárez, A.D. (2002). Cuantificación y valoración económica del servicio ambiental almacenamiento de carbono en sistemas agroforestales de café en la comarca Yassica Sur, Matagalpa, Nicaragua. Centro Agronómico Tropical de Investigación y Enseñanza (CATIE) Turrialba, Costa Rica. $117 \mathrm{p}$.

Van Asten, P.J.A., Wairegi, L.W.I., Mukasa, D., \& Uringi, N.O. (2011). Agronomic and economic benefits of coffee-banana intercropping in Uganda's smallholder farming systems. Agricultural Systems, 104, 326-334.

Vargas, T., \& Samayoa, S. (2009). Mecanismo de desarrollo limpio, conceptos básicos. Guía para formulación y presentación de proyectos. Primera edición, Honduras, 57 p.

Walkley, A., \& Black, C.A. (1934). An examination of the Degtajareff's method for determining soil organic matter and a proposed modification of the chromic acid titration method. Soil Science, 37, 29-38. 